\title{
A NEW GENERALIZATION OF DELAYED FEEDBACK CONTROL
}

\author{
ÖMER MORGÜL \\ Department of Electrical Engineering, \\ Bilkent University, \\ Ankara, Turkey \\ morgul@ee.bilkent.edu.tr
}

Received October 15, 2007; Revised May 19, 2008

\begin{abstract}
In this paper, we consider the stabilization problem of unstable periodic orbits of one-dimensional discrete time chaotic systems. We propose a novel generalization of the classical delayed feedback law and present some stability results. These results show that for period 1 all hyperbolic periodic orbits can be stabilized by the proposed method; for higher order periods the proposed scheme possesses some inherent limitations. However, some more improvement over the classical delayed feedback scheme can be achieved with the proposed scheme. The stability proofs also give the possible feedback gains which achieve stabilization. We will also present some simulation results.
\end{abstract}

Keywords: Chaotic systems; chaos control; delayed feedback; pyragas controller.

\section{Introduction}

It has been well known that the mathematical models of many physical systems exhibit chaotic behavior, see e.g. [Chen \& Dong, 1998]. As a result, the possibility of controlling chaos has attracted a great deal of attention among scientists from various disciplines including physicists over the past decade, see e.g. [Chen \& Dong, 1998; Boccaletti et al., 2000], and the references therein.

Chaotic systems possess strange attractors and usually many unstable periodic orbits are embedded in them, see e.g. [Devaney, 1987]. One of the control problems is to stabilize some of these periodic orbits hence forcing the chaotic systems to exhibit regular behavior, see e.g. [Chen \& Dong, 1998]. A remarkable result first given in [Ott et al., 1990] proves that some of these orbits can be stabilized by using small external forces. Following the seminal work of [Ott et al., 1990], various other control methods have been proposed for the cited problem. Among these, the Delayed Feedback Control (DFC) scheme first proposed in [Pyragas, 1992] has received attention due to its various attractive features. This scheme has also been used in various applications, see e.g. [Ishii et al., 1997; Pyragas, 2001; Morgül, 2003, 2006], and the references therein. As it is shown in [Ushio, 1996; Nakajima, 1997; Morgül, 2003, 2005a; Zhu \& Tian, 2005b] the classical DFC has certain inherent limitations, i.e. it cannot stabilize certain periodic orbits. This limitation is known as the odd-number limitation of the DFC schemes. More precisely, the question is whether a hyperbolic and unstable periodic orbit with an odd number of real unstable modes can be stabilized with DFC or not. Early investigations indicated that this property holds for discrete-time systems, see e.g. [Ushio, 1996], and later an extension of this result to continuous time case was claimed in e.g. [Nakajima, 1997]. At this point, we must comment on a recent result presented in [Fiedler et al., 2007], which showed clearly that under certain cases, odd number limitation property does not hold. An autonomous continuous time system is considered in [Fiedler et al., 2007] 
and by way of constructing a feedback gain it was shown that DFC can stabilize a hyperbolic unstable orbit which has an odd number of real unstable modes, hence clearly refutes the claim given in [Nakajima, 1997] for at least autonomous continuous time systems. However, whether such a refutation can be extended to nonautonomous continuous time case or not is still an open question, see e.g. [Höhne et al., 2007]. Although the subject is still open and deserves further investigation, we note that the limitation of DFC stated above holds for discrete time case, see e.g. [Ushio, 1996; Morgül, 2003, 2005a; Zhu \& Tian, 2005b].

To overcome the limitations of classical DFC scheme, various modifications have been proposed, see e.g. [Socolar et al., 1994; Kittel et al., 1995; Pyragas, 1995; Bleich \& Socolar, 1996; Vieira \& Lichtenberg, 1996; Schuster \& Stemmler, 1997; Nakajima \& Ueda, 1998a; Pyragas, 2001; Hino et al., 2002], and the references therein. One of these schemes is the so-called periodic, or oscillating feedback, see [Schuster \& Stemmler, 1997], and is known that it eliminates the limitations of classical DFC for period $T=1$ case. This scheme can be generalized to the case $T>1$ in various ways, and two such generalizations are given in [Morgül, 2005b, 2006]; it has been shown in these references that any hyperbolic periodic orbit can be stabilized with these schemes. Another modification is the so-called extended DFC (EDFC), see [Socolar et al., 1994]. This scheme is then analyzed and various modifications have been proposed, see e.g. [Pyragas, 1995; Bleich \& Socolar, 1996; Vieira \& Lichtenberg, 1996; Ishii et al., 1997; Pyragas, 2001; Harrington \& Socolar, 2004], and the references therein. It has also been shown that EDFC also has inherent limitations similar to the DFC, see [Ishii et al., 1997; Nakajima \& Ueda, 1998b]. In [Vieira \& Lichtenberg, 1996], a nonlinear version of EDFC has been proposed and it was shown that an optimal version of this scheme becomes quite simple, e.g. the dimension of the phase space of the controlled system becomes the same as that of the original system for $T=1$. In this paper we will propose a scheme which is related to the optimal control law proposed in [Vieira \& Lichtenberg, 1996] for the case $T=1$ and we then generalize the proposed scheme for $T>1$.

This paper is organized as follows. In Sec. 2 we will outline the basic problem. In Sec. 3 we will propose a new generalization of the DFC scheme and in Sec. 4 we will provide some stability results.
In Sec. 5 we will provide some simulation results and finally we will give some concluding remarks.

\section{Problem Statement}

Let us consider the following discrete-time system

$$
x(k+1)=f(x(k)),
$$

where $k=1,2 \ldots$ is the discrete time index, $x \in$ $\mathbf{R}, f: \mathbf{R} \rightarrow \mathbf{R}$ is an appropriate function, which is assumed to be differentiable wherever required. We assume that the system given by (1) possesses a period $T$ orbit characterized by the set

$$
\Sigma_{T}=\left\{x_{1}^{*}, x_{2}^{*}, \ldots, x_{T}^{*}\right\},
$$

i.e. for $x(1)=x_{1}^{*}$, the iterates of (1) yields $x(2)=$ $x_{2}^{*}, \ldots, x(T)=x_{T}^{*}, x(k)=x(k-T)$ for $k>T$.

Let $x(\cdot)$ be a solution of (1). To characterize the convergence of $x(\cdot)$ to $\Sigma_{T}$, we need a distance measure, which is defined as follows. For $x_{i}^{*}$, we will use circular notation, i.e. $x_{i}^{*}=x_{j}^{*}$ for $i=j(\bmod (T))$. Let us define the following indices $(j=1, \ldots, T)$ :

$$
d_{k}(j)=\sqrt{\sum_{i=0}^{T-1}\left|x(k+i)-x_{i+j}^{*}\right|^{2}} .
$$

We then define the following distance measure

$$
d\left(x(k), \Sigma_{T}\right)=\min \left\{d_{k}(1), \ldots, d_{k}(T)\right\} .
$$

Clearly, if $x(1) \in \Sigma_{T}$, then $d\left(x(k), \Sigma_{T}\right)=0, \forall k$. Conversely if $d\left(x(k), \Sigma_{T}\right)=0$ for some $k_{0}$, then it remains 0 and $x(k) \in \Sigma_{T}$, for $k \geq k_{0}$. We will use $d\left(x(k), \Sigma_{T}\right)$ as a measure of convergence to the periodic solution given by $\Sigma_{T}$.

Let $x(\cdot)$ be a solution of (1) starting with $x(1)=x_{1}$. We say that $\Sigma_{T}$ is (locally) asymptotically stable if there exists an $\varepsilon>0$ such that for any $x(1) \in \mathbf{R}$ for which $d\left(x(1), \Sigma_{T}\right)<\varepsilon$ holds, we have $\lim _{k \rightarrow \infty} d\left(x(k), \Sigma_{T}\right)=0$. Moreover if this decay is exponential, i.e. the following holds for some $M \geq 1$ and $0<\rho<1,(k>1)$ :

$$
d\left(x(k), \Sigma_{T}\right) \leq M \rho^{k} d\left(x(1), \Sigma_{T}\right),
$$

then we say that $\Sigma_{T}$ is (locally) exponentially stable.

To stabilize the periodic orbits of (1), let us apply the following control law:

$$
x(k+1)=f(x(k))+u(k)
$$

where $u(\cdot)$ is the control input. In classical DFC, the following feedback law is used $(k>T)$ :

$$
u(k)=K(x(k)-x(k-T)),
$$


where $K \in \mathbf{R}$ is a constant gain to be determined. It is known that the scheme given above has certain inherent limitations, see e.g. [Ushio, 1996]. For $\Sigma_{T}$, let us set $a_{i}=f^{\prime}\left(x_{i}^{*}\right)$. It can be shown that $\Sigma_{T}$ cannot be stabilized with this scheme if $a=\prod_{i=1}^{T} a_{i}>$ 1, see e.g. [Ushio, 1996; Morgül, 2003], and a similar condition can be generalized to higher dimensional case [Nakajima, 1997; Hino et al., 2002; Morgül, 2005a; Zhu \& Tian, 2005b]. A set of necessary and sufficient conditions to guarantee exponential stabilization can be found in [Morgül, 2003] for one-dimensional and in [Morgül, 2005a] for higher dimensional cases. By using these results one can find a suitable gain $K$ when stabilization is possible. We note that even if $a<1$, the stabilization is not guaranteed for classical DFC scheme. For example, for one-dimensional and period 1 case, stabilization is only possible when $-3<a<1$, see e.g. [Ushio, 1996; Morgül, 2003].

\section{A Different Generalization of DFC}

To overcome the basic limitations of DFC, various modifications have been proposed as mentioned in the introduction. Among these, the EDFC scheme first proposed in [Socolar et al., 1994] and its nonlinear version proposed in [Vieira \& Lichtenberg, 1996] deserve special attention. In the sequel we will propose a scheme which is related to the optimal version of the scheme proposed in [Vieira \& Lichtenberg, 1996] for the case $T=1$ and then generalize it to the case $T>1$.

To motivate our approach, let $\Sigma_{1}=\left\{x_{1}^{*}\right\}$ be a period 1 orbit of (1) (i.e. fixed point of $f: \mathbf{R} \rightarrow \mathbf{R}$ ), and consider the controlled system given by (6). Instead of the DFC scheme given by (7), let us propose the following law:

$$
u(k)=\frac{K}{K+1}(x(k)-f(x(k))),
$$

where $K \in \mathbf{R}$ is a constant gain to be determined. We could also use the gain as $\hat{K}=K /(K+1)$, however the suitability of the form of gain $K$ as given in (8) will become clear in the sequel, see e.g. Remark 2 below. Obviously we assume $K \neq-1$. If we use (8) in (6), we obtain:

$$
\begin{aligned}
x(k+1) & =f(x(k))+\frac{K}{K+1}(x(k)-f(x(k))) \\
& =\frac{1}{K+1} f(x(k))+\frac{K}{K+1} x(k) .
\end{aligned}
$$

First note that on $\Sigma_{1}$, we have $u(k)=0$; moreover if $x(k) \rightarrow \Sigma_{1}$ (i.e. $\Sigma_{1}$ becomes asymptotically stable) we have $u(k) \rightarrow 0$ as well. Hence, the scheme proposed in (8) enjoys similar properties of DFC. Moreover, note that unlike the DFC scheme, since the delay term does not appear in the control term for the period 1 case, the dimension of the phase space of the controlled system given by (9) is 1, i.e. (9) is defined on $\mathbf{R}$. On the other hand, for $T=1$, if one uses the classical DFC scheme as given by (6) and (7), by defining the standard variables $x_{1}(k)=x(k-1)$ and $x_{2}(k)=x(k)$, see e.g. [Khalil, 2002], one obtains $x_{1}(k+1)=x_{2}(k)$, $x_{2}(k+1)=f\left(x_{2}(k)\right)+K\left(x_{2}(k)-x_{1}(k)\right)$, which shows that the actual dimension of the phase space is 2. This argument may be considered as an advantage of the proposed scheme over the classical DFC and EDFC. Later we will show that for the higher order periodic orbits, a similar conclusion holds.

Remark 1. At this point if we compare the form given by (9) with the optimal control law given in [Vieira \& Lichtenberg, 1996], we see that they have the same form except for that of the constant gain. If in (9) we set $\hat{K}=K /(K+1)$, with the gain $\hat{K}$, we obtain the form of the optimal control law given by Eq. (22) in [Vieira \& Lichtenberg, 1996]. A similar control law is also used in [Ushio \& Yamamoto, 1999] for period 1 case, see Eq. (5) of the latter reference. Hence for period 1 case, our proposed method is similar to those as given in [Vieira \& Lichtenberg, 1996] and [Ushio \& Yamamoto, 1999]. However, in the sequel we will show that for higher order periods (i.e. $T>1$ ), our proposed method is quite different than those proposed in these references.

Remark 2. The scheme given above has an interesting relation with the classical DFC scheme. To see that, if we multiply (9) with $K+1$, after simplification we obtain:

$$
x(k+1)=f(x(k))+K(x(k)-x(k+1)) .
$$

If we compare (10) with (6), we see that they become similar if we use the following equation for $u(k)$ :

$$
u(k)=K(x(k)-x(k+1)) .
$$

However, this is only a mathematical similarity since $u(k)$ given by (11) is not implementable as a control law. Note that the control schemes given in [Vieira \& Lichtenberg, 1996] and [Ushio \& Yamamoto, 1999] for period 1 case also have this 
property. Nevertheless, we think that this mathematical equivalence is interesting at least for two reasons. First, it shows the mathematical relation of the proposed scheme with the classical DFC, see (11) and (7). Secondly, the form given by (11), although not meaningful from implementation point of view, could help us to generalize this scheme for higher order periodic orbits. This will be done in the sequel.

For the stability analysis, set $\Sigma_{1}=\left\{x_{1}^{*}\right\}, a=$ $a_{1}=f^{\prime}\left(x_{1}^{*}\right)$. By using linearization, (9) and the classical Lyapunov stability analysis, we see that $\Sigma_{1}$ is exponentially stable for (9) if and only if

$$
\left|\frac{K+a}{K+1}\right|<1
$$

Clearly, if $a \neq 1$, then any $\Sigma_{1}$ can be stabilized by choosing $K$ appropriately to satisfy (12). Hence the limitation of DFC is eliminated greatly by the proposed approach. It appears that the only restriction that remains (i.e. $a \neq 1$ ) is quite inherent and appears in [Morgül, 2005b], and [Morgül, 2006] as well; see also [Harrington \& Socolar, 2004] for the same restriction, where it is referred to as a stationary mode. By using the arguments given in these latter references, we can state that all hyperbolic fixed points can be stabilized by the proposed scheme.

\section{General Case and Stability Analysis}

In the sequel, we will generalize the control law given by (9) to $T=m$ case. To motivate our analysis, first let us consider the case $T=2$, in which case we propose the following control law:

$$
u(k)=\frac{K}{K+1}(x(k-1)-f(x(k)),
$$

where $K \in \mathbf{R}$ is a constant gain to be determined. If we use (13) in (6), we obtain:

$$
\begin{aligned}
x(k+1) & =f(x(k))+\frac{K}{K+1}(x(k-1)-f(x(k))) \\
& =\frac{1}{K+1} f(x(k))+\frac{K}{K+1} x(k-1) .
\end{aligned}
$$

Note that, the dimension of the phase space for the controlled system given by (14) is 2 , whereas for period 2 case, the dimension of the phase space of the classical DFC given by (6) and (7) is 3.
Remark 3. As mentioned in Remark 2, the scheme given above has an interesting relation with the classical DFC scheme. To see that, if we multiply (14) with $K+1$, after simplification we obtain:

$x(k+1)=f(x(k))+K(x(k-1)-x(k+1))$.

If we compare (15) with (6), we see that they become similar if we use the following equation for $u(k)$ :

$$
u(k)=K(x(k-1)-x(k+1)) .
$$

However, this is only a mathematical similarity since $u(k)$ given by (16) is not implementable as a control law. Nevertheless, this form may help us to generalize the proposed control scheme to the case $T>1$.

Let $\Sigma_{2}=\left\{x_{1}^{*}, x_{2}^{*}\right\}$ be a period 2 orbit of (1) and let us set

$$
a_{1}=f^{\prime}\left(x_{1}^{*}\right), \quad a_{2}=f^{\prime}\left(x_{2}^{*}\right), \quad a=a_{1} a_{2} .
$$

For stability analysis, we will follow the methodology given in [Morgül, 2003, 2005a]. Let us define $x_{1}(k)=x(k-1), x_{2}(k)=x(k)$ and $z=\left(\begin{array}{ll}x_{1} & x_{2}\end{array}\right)^{T}$ where the superscript $T$ denotes the transpose. Let us define a map $F: \mathbf{R}^{2} \rightarrow \mathbf{R}^{2}$ as $F(z)=\left(\begin{array}{ll}x_{2} & Y_{1}\end{array}\right)^{T}$ where $Y_{1}=(1 /(K+1)) f\left(x_{2}\right)+(K /(K+1)) x_{1}$. Clearly we have $F^{2}(z)=\left(\begin{array}{ll}Y_{1} & Y_{2}\end{array}\right)^{T}$ where $Y_{2}=$ $(1 /(K+1)) f\left(Y_{1}\right)+(K /(K+1)) x_{2}$. Let us consider the system:

$$
z(k+1)=F^{2}(z(k)) .
$$

Consider the fixed points of (18), i.e. $F^{2}\left(z^{*}\right)=z^{*}$ where $z^{*}=\left(\begin{array}{ll}x_{1}^{*} & x_{2}^{*}\end{array}\right)^{T}$. Solving the fixed point equation, after simple calculations we obtain $x_{2}^{*}=f\left(x_{1}^{*}\right)$ and $x_{1}^{*}=f\left(x_{2}^{*}\right)$. Hence the fixed point $z^{*}$ of $(18)$ corresponds to a period 2 orbit $\Sigma_{2}$ of (1), and vice versa. Therefore, for the stability of $\Sigma_{2}$, we study the stability of the corresponding fixed point $z^{*}$ for the map $F^{2}$. This can be done by standard linearization. The Jacobian $J_{2}=\left(\partial F^{2} / \partial z\right) \mid \Sigma_{2}$ can easily be obtained as:

$$
J_{2}=\left[\begin{array}{cc}
\frac{K}{K+1} & \frac{a_{1}}{K+1} \\
\frac{K a_{2}}{(K+1)^{2}} & \frac{K}{K+1}+\frac{a}{(K+1)^{2}}
\end{array}\right] .
$$

For stability analysis, we may calculate the characteristic polynomial $p_{2}(\lambda)=\operatorname{det}\left(\lambda I-J_{2}\right)$ where $I$ 
is an identity matrix with appropriate dimensions. The latter can easily be found as

$$
p_{2}(\lambda)=\left(\lambda-\frac{K}{K+1}\right)^{2}-\frac{a}{(K+1)^{2}} \lambda .
$$

At this point we can generalize the control law given by (9) to $T=m$ case. By following the ideas given above, we propose the following control law:

$$
u(k)=\frac{K}{K+1}(x(k-m+1)-f(x(k)),
$$

where $K \in \mathbf{R}$ is a constant gain to be determined. If we use (21) in (6), we obtain:

$$
\begin{aligned}
x(k+1) & =f(x(k))+\frac{K}{K+1}(x(k-m+1)-f(x(k)) \\
& =\frac{1}{K+1} f(x(k))+\frac{K}{K+1} x(k-m+1) .
\end{aligned}
$$

Note that, the dimension of the phase space for the controlled system given by $(22)$ is $m$, whereas for period $m$ case, the dimension of the phase space of the classical DFC given by (6) and (7) is $m+1$.

Remark 4. As mentioned in Remarks 2 and 3, the scheme given above has an interesting relation with the classical DFC scheme. To see that, if we multiply (22) with $K+1$, after simplification we obtain:

$$
x(k+1)=f(x(k))+K(x(k-m+1)-x(k+1)) .
$$

If we compare (23) with (6), we see that they become similar if we use the following equation for $u(k)$ :

$$
u(k)=K(x(k-m+1)-x(k+1)) .
$$

However, this is only a mathematical similarity since $u(k)$ given by $(24)$ is not implementable as a control law.

For stability analysis, we will follow the methodology given in [Morgül, 2003, 2005a]. As before, let us define $x_{i}(k)=x(k-m+i), i=$ $1,2, \ldots, m$ and $z=\left(x_{1} \cdots x_{m}\right)^{T}$. Let us define

$$
\begin{array}{r}
Y_{i}=\frac{1}{K+1} f\left(Y_{i-1}\right)+\frac{K}{K+1} x_{i}, \quad Y_{0}=x_{m}, \\
i=1,2, \ldots, m .
\end{array}
$$

Let us define the map $F: \mathbf{R}^{m} \rightarrow \mathbf{R}^{m}$ as $F(z)=\left(x_{2} x_{3} \cdots x_{m} Y_{1}\right)^{T}$. Clearly we have $F^{m}=$ $\left(Y_{1} Y_{2} \cdots Y_{m}\right)^{T}$. Now, consider the map

$$
z(k+1)=F^{m}(z(k)) .
$$

Now consider the fixed points of $(26)$, i.e. $F^{m}\left(z^{*}\right)=$ $z^{*}$ where $z^{*}=\left(x_{1}^{*} x_{2}^{*} \ldots x_{m}^{*}\right)^{T}$. Clearly we will have $x_{i}^{*}=Y_{i}$ where $i=1,2, \ldots, m$ and $Y_{i}$ are given by (25). Solving these equations we easily obtain $x_{i+1}^{*}=f\left(x_{i}^{*}\right), i=1,2, \ldots, m-1$ and $x_{1}^{*}=f\left(x_{m}^{*}\right)$. This shows that a fixed point $z^{*}$ of (26) corresponds to a period $m$ orbit $\Sigma_{m}$ of (1), and vice versa. Therefore for the stability of $\Sigma_{m}$, we can study the stability of the fixed point $z^{*}$ of (26). This can be done by standard linearization, i.e. by finding the Jacobian $J_{m}=\left(\partial F^{m} / \partial z\right) \mid \Sigma_{m}$. Clearly we have $J_{m}(i, j)=\left(\partial Y_{i} / \partial x_{j}\right) \mid \Sigma_{m}$. By using (25), after straightforward calculations we obtain:

$$
\begin{gathered}
\frac{\partial Y_{i}}{\partial x_{i}}=\frac{K}{K+1}, \quad i=1,2, \ldots, m-1, \\
\frac{\partial Y_{m}}{\partial x_{m}}=\frac{K}{K+1}+\frac{a}{(K+1)^{m}}, \\
\frac{\partial Y_{i}}{\partial x_{j}}=\frac{a_{i-1}}{K+1} \frac{\partial Y_{i-1}}{\partial x_{j}}, \quad i, j=1,2, \ldots, m, \quad i \neq j,
\end{gathered}
$$

where by convention we have $a_{0}=a_{m}, Y_{0}=Y_{m}$. For stability analysis, we need the characteristic polynomial of $J_{m}$, which is given in the following Theorem.

Theorem 1. Let $\Sigma_{m}$ given by (2) be a period $T=$ $m$ orbit of (1) and set $a_{i}=f^{\prime}\left(x_{i}\right), i=1,2, \ldots, m$, $a=\prod_{i-1}^{m} a_{i}$. Consider the Jacobian $J_{m}$ given by (25)-(28). Then for $m \geq 1$ we have:

$$
\begin{aligned}
p_{m}(\lambda) & =\operatorname{det}\left(\lambda I-J_{m}\right) \\
& =\left(\lambda-\frac{K}{K+1}\right)^{m}-\frac{a}{(K+1)^{m}} \lambda^{m-1},
\end{aligned}
$$

where $I$ is an identity matrix with appropriate dimensions.

Proof. This result can easily be shown either by using direct calculation or by using a mathematical induction. The calculations are straightforward but rather lengthy and hence are omitted here.

We say that a polynomial is Schur stable if all of its roots are inside the unit disc of the complex plane, i.e. have magnitude less than unity. Hence the asymptotic stability of the fixed points of (26) hence the asymptotic stability of $\Sigma_{m}$ for (6) and (21) could be analyzed by considering the Schur stability of $p_{m}(\lambda)$. Moreover note that the exponential stability is equivalent to Schur stability, see [Khalil, 2002]. By using these, we can state our next result. 
Theorem 2. Let $\Sigma_{m}$ given by (2) be a period $T=$ $m$ orbit of (1) and set $a_{i}=f^{\prime}\left(x_{i}\right), i=1,2, \ldots, m$, $a=\prod_{i-1}^{m} a_{i}$. Consider the control scheme given by (6) and (21). Then:

(i) $\Sigma_{m}$ is exponentially stable if and only if $p_{m}(\lambda)$ given by (29) is Schur stable. This condition is only sufficient for asymptotic stability.

(ii) If $p_{m}(\lambda)$ has at least one unstable root, i.e. outside the unit disc, then $\Sigma_{m}$ is unstable as well.

(iii) If $p_{m}(\lambda)$ is marginally stable, i.e. has at least one root on the unit disc while the rest of the roots are inside the unit disc, then the proposed method to test the stability of $\Sigma_{m}$ is inconclusive.

Proof. The proof of this Theorem easily follows from standard Lyapunov stability arguments, see e.g. [Khalil, 2002], and [Morgül, 2003, 2005a] for similar arguments.

At this point several comments are in order.

Remark 5. For $m=1$, we have $p_{1}(\lambda)=\lambda-$ $((K+a) /(K+1))$. Hence, according to Theorem $2, \quad \Sigma_{1}$ is exponentially stable if and only if $|((K+a) /(K+1))|<1$, which confirms (12). Obviously such a gain $K$ can always be found if and only if $a \neq 1$. Hence for $m=1$, the proposed scheme eliminates the inherent limitations of DFC. As in periodic feedback case, by using the arguments given in [Morgül, 2005b, 2006], a periodic extension of this result to stabilize arbitrary $\Sigma_{m}$ could be studied. However, this approach is beyond the scope of the present work.

Remark 6. Let $m>1$. First note that for $m=2$, the polynomials $p_{m}(\lambda)$ given by (29) and (20) coincide. For an arbitrary $m>1$, the Schur stability of $p_{m}(\lambda)$ given by (29) can be checked by applying the SchurCohn criterion, or equivalently the Jury test, see [Elaydi, 1996]. These tests give some necessary and sufficient conditions in terms of the coefficients of $p_{m}(\lambda)$. Since these coefficients depend on the gain $K$, these tests result in a finite set of inequalities in terms of $K$, hence by solving these one may find the range of $K$ when the stabilization is possible. See e.g. [Morgül, 2003] for a similar approach.

Remark 7. The polynomial $p_{m}(\lambda)$ given by (29) depends only on $K$ and $a$. However, in DFC, a similar polynomial depends on all $a_{i}$, see [Morgül, 2003]. As a result, some periodic orbits which cannot be stabilized by DFC may be stabilized with the proposed approach, see simulation examples.

Remark 8. The proposed scheme also has the same inherent limitations of DFC and EDFC for $m>1$. To see this, let $\lambda_{i}$ be the roots of $p_{m}(\lambda)$ given by (29). Obviously, for stability we require $\prod_{i=1}^{m}\left|\lambda_{i}\right|<$ 1 , which requires $|K /(K+1)|<1$ for $m>1$. The last inequality implies $K>-0.5$, hence $K+1>0$ in the stable case. A necessary condition for Schur stability of $p_{m}(\lambda)$ is $p_{m}(1)>0$, see [Elaydi, 1996]. The latter results in $1-a>0$. This shows that for the case $m>1$, stabilization is not possible for $a>1$, similar to DFC and EDFC schemes.

Remark 9. Another necessary condition for the Schur stability of $p_{m}(\lambda)$ is $(-1)^{m} p_{m}(-1)>0$, see [Elaydi, 1996]. By using (29) and the fact that $K+1>0$ for stability, we obtain:

$$
K>K_{c r}=-0.5+0.5(-a)^{1 / m} .
$$

Note that if $\Sigma_{m}$ is unstable, then we must have $|a| \geq 1$, and if stabilizable with the proposed scheme then we need $a<1$. Hence if $\Sigma_{m}$ is unstable and is stabilizable by our scheme, we need $a<-1$, which implies $-a>1$ in our case. It can be shown that if $m=2$ and $a<-1$, then $\Sigma_{2}$ is always stabilizable with the proposed scheme and in this case (30) gives the exact range of stabilizing gains for our scheme. Note that many $\Sigma_{2}$ cannot be stabilizable with DFC, see e.g. [Morgül, 2003].

Remark 10. Let $m \geq 3$. It is clear from (29) that if $|a|$ is sufficiently small, $p_{m}(\lambda)$ is always Schur stable. In other words, let $m>2$, and $a<-1$. For any $m$, there exists an $a_{m c r}>0$ such that for $|a|<a_{m c r}$, the stabilization is possible with the proposed scheme. After some extensive numerical calculations, we found that $a_{3 c r}=27, a_{4 c r}=15$, $a_{5 c r}=11.5, a_{6 c r}=9.8$. It seems that $a_{m c r} \rightarrow 1$ as $m \rightarrow \infty$. However, this point requires further justification. (Note that for $|a|<1, \Sigma_{m}$ is already stable).

Remark 11. In our simulations we observed that for a given $a<-1$, if for $K=K_{c r}$ given by $(30), p_{m}(\lambda)$ is marginally stable, see Theorem 2 , then one can find an upper bound $K_{m}$ such that stabilization is possible for $K_{c r}<K<K_{m}$. However, this is just an observation and requires further justification.

Remark 12. At this point we may compare the control law proposed here with the control laws 
given in [Vieira \& Lichtenberg, 1996] and [Ushio \& Yamamoto, 1999]. As stated in Remark 1, for $T=1$ case, these control laws are similar. However, for $T>1$, these control laws are quite different. In particular, for $T=m>1$ case, the control law given in [Vieira \& Lichtenberg, 1996] becomes (see Eq. (31) of the latter reference):

$x(k+m)=f^{m}(x(k))+\hat{K}\left(x(k)-f^{m}(x(k))\right)$,

and for the problem considered in this paper the control law proposed in [Ushio \& Yamamoto, 1999] becomes (see Eq. (6) of the latter reference)

$$
x(k+1)=f(x(k))-\hat{K}\left(x(k)-f^{m}(x(k))\right) .
$$

By comparing (22) with (31) and (32), we see that these schemes are quite different for $T=m>1$. While in (22), a delay term is present, such a term does not appear in (31) and (32). The usage of $f^{m}(x(k))$ in (31) and (32) may pose some computational problems especially for higher order periodic orbits. Moreover, although these methods are proposed for an arbitrary $m$, the stability analysis are given for $m=1$ case; the extension of these stability results to case $m>1$ is not clear. We note that this is an interesting topic which requires and deserves further investigation. Another difference between the method proposed here and the ones mentioned above is related to the periodic orbits of the controlled and uncontrolled systems. Now let $\Sigma_{m}$ as given by (2) be a period $m$ orbit of the uncontrolled system given by (1). It follows easily that $\Sigma_{m}$ is also a period $m$ orbit of the controlled system given by (6) and (7). Converse of this property also holds, i.e. any period $m$ orbit $\Sigma_{m}$ of the controlled system given by (6) and (7) is also a period $m$ orbit of the uncontrolled system given by (1). This is a general property of DFC scheme for discrete time systems and can be shown easily since the control input given by (7) vanishes for any $K$ on the periodic orbit. This property also holds for our scheme, and the proof of this statement follows easily from the mathematically equivalent form of $u(k)$ given by (24); obviously with some straightforward algebra this property can also be proven by using the actual controlled system equations given by (22). However, this equivalence fails for the system given by e.g. (32). More precisely, for some $\hat{K}$, the system given by (32) may possess a periodic orbit which is not a periodic orbit of the uncontrolled system given by (1) for case $m>1$. As an example, let $m=2$, and let $x_{*}$ be a point which satisfies $x_{*} \neq f\left(x_{*}\right)$ and $x_{*} \neq f^{2}\left(x_{*}\right)$. Now choose $\hat{K}=\hat{K}_{*}=\left(f\left(x_{*}\right)-x_{*}\right) /\left(x_{*}-f^{2}\left(x_{*}\right)\right)$. Clearly for $m=2$, if we choose $\hat{K}=\hat{K}_{*}$ as given above, for $x(k)=x_{*}$, we have $x(k+1)=x_{*}$, hence $x_{*}$ becomes a fixed point of (32), which is not a fixed point of (1). Moreover, depending on $f$ and the chosen $x_{*}$, it may be possible to stabilize both $x_{*}$ and an unstable fixed point of (1) for the system given by (32) under certain conditions by using the gain $\hat{K}_{*}$ as given above. This is an interesting feature of (32) and requires further investigation.

Remark 13. Note that the feedback law given by (6)-(7) is linear, whereas the feedback law given by (6) and (21) is nonlinear. This point might be considered as a drawback since the linear control laws are simpler than the nonlinear ones, see e.g. [Harrington \& Socolar, 2004]. However, to stabilize an unstable periodic orbit for a nonlinear system is a difficult task and in general for such complex problems many research results presented in the literature in recent years resort to nonlinear feedback laws, see e.g. [Khalil, 2002]. For example, a wellknown technique called computed torque in robotics and related areas relies on the usage of the nonlinear vector field (i.e. $f(x)$ in our case), see e.g. [Khalil, 2002]. Another well-known technique called feedback linearization relies on a nonlinear transformation and a nonlinear feedback law, and in determining the appropriate controller the knowledge of the vector field (i.e. $f(x)$ in our case) is essential, see e.g. [Khalil, 2002]. Such nonlinear techniques are also used in the literature for both the control of chaotic systems and for the problem considered above, see e.g. [Fuh \& Tung, 1995; Vieira \& Lichtenberg, 1996; Ushio \& Yamamoto, 1998; Liu \& Tan, 1998; Ushio \& Yamamoto, 1999; Solak et al., 2001; Hino et al., 2002; Fradkov \& Evans, 2005; Zhu \& Tian, 2005a, 2005b; Zhu \& Tian, 2006], and the references therein. Hence, from this point, applying a nonlinear control law to a nonlinear system to solve a complex problem should be considered as a natural approach. Also note that the control law given by (21) is not based on cancelation, contrary to some existing schemes, see e.g. [Zhu \& Tian, 2005a]. One may also argue that to implement the control law given by (21), one needs the knowledge of $f(\cdot)$, whereas this is not required to implement (7). First, the knowledge of $f(\cdot)$ is also required in many nonlinear control techniques, see e.g. the references cited above. Secondly, although in (7) the knowledge of $f(\cdot)$ is not explicitly required, to 
determine whether a stabilizing gain $K$ exists or not, and in affirmative case to determine the stabilizing gains one needs the knowledge of $f(\cdot)$, see e.g. [Ushio, 1996; Morgül, 2003, 2005a]. Note that if $f(\cdot)$ is known and if $x$ is available, as in (7), then constructing $f(x)$ will not pose a problem.

\section{Simulation Results}

We consider the well-known logistic map given as

$$
x(k+1)=r x(k)(1-x(k)) .
$$

We will not consider case $T=1$ since it can also be stabilized by EDFC. Let us consider case $T=2$. For $r=4$, it is well-known that (33) has periodic orbits of all orders, and in particular it

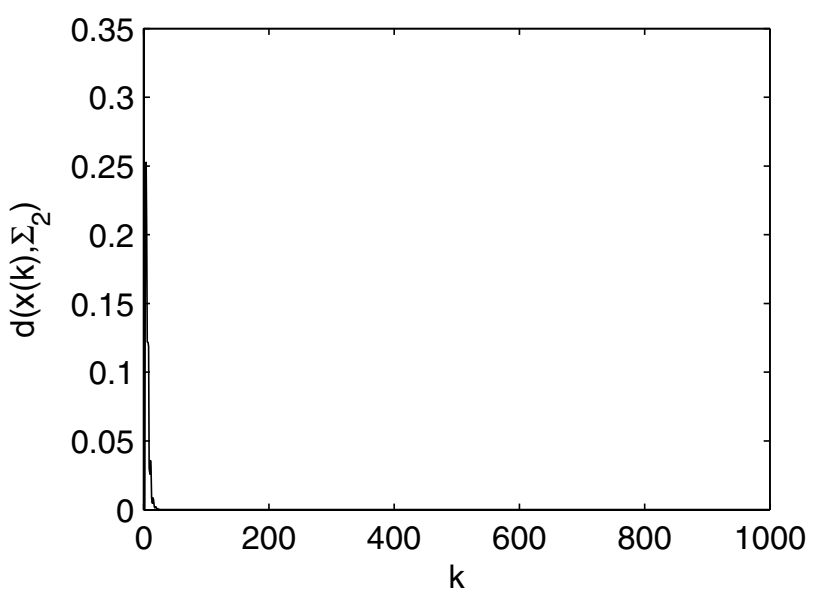

(a)

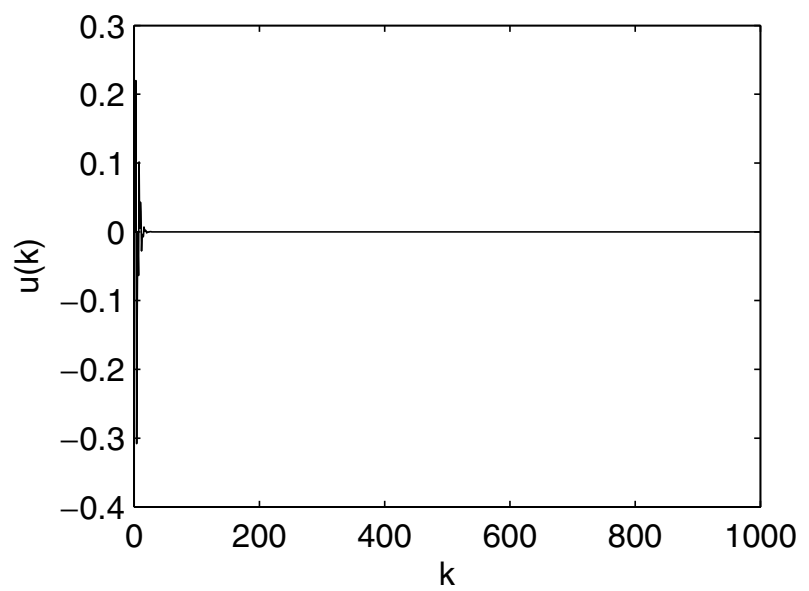

(c) has a period 2 orbit given by $\Sigma_{2}=\left\{x_{1}^{*} x_{2}^{*}\right\}$ with $x_{1}^{*}=0.90450849718747, x_{2}^{*}=0.34549150281253$, with $a=-4$. By Remark $5, \Sigma_{2}$ is stabilizable with any $K>0.5$. In Fig. 1, we show the simulation result of a typical case with $K=1$; the initial condition is chosen as $x(0)=0.6$. In Fig. 1(a), we show $d\left(x(k), \Sigma_{2}\right)$ versus $k$, and as can be seen the decay is exponential. The $x(k-1)$ versus $x(k)$ plot in Fig. 1(b) is plotted for $k \geq 500$. As can be seen from these figures, the solutions converge to the period 2 orbit characterized by $\Sigma_{2}$. The required input $u(k)$ given by (21) is given in Fig. 1(c). As can be seen, $u(k)$ is quite small and $\rightarrow 0$ as $k \rightarrow \infty$. Finally Fig. $1(d)$ shows $x(k)$ versus $k$ for $980 \leq k \leq 1000$. Note that this orbit, in fact none of the period 2 orbits of (33) with $r>3.77$, can be stabilized by DFC, see [Morgül, 2003].

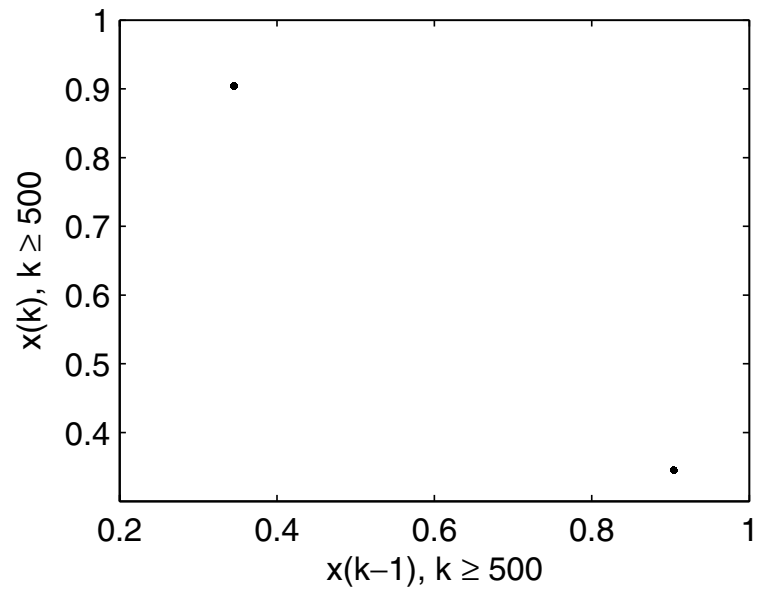

(b)

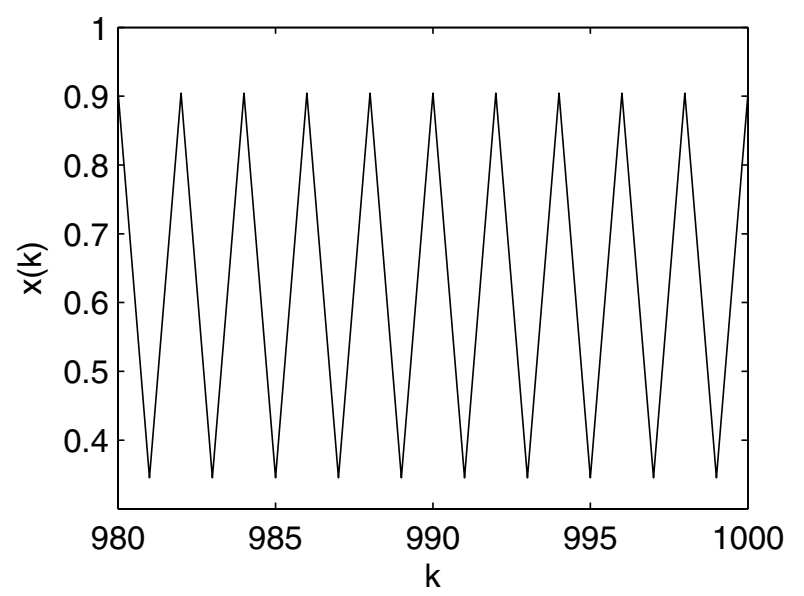

(d)

Fig. 1. Proposed scheme applied to logistic map, $r=4, T=2$, (a) $d\left(x(k), \Sigma_{2}\right)$ versus $k$, (b) $x(k-1)$ versus $x(k)$ for $k \geq 500$, (c) $u(k)$ versus $k,(\mathrm{~d}) x(k)$ versus $k$ for $980 \leq k \leq 1000$. 
Now let us choose $r=4$ and $T=3$. The system (33) has a period 3 orbit given by $\Sigma_{2}=$ $\left\{x_{1}^{*} x_{2}^{*} x_{3}^{*}\right\}$ with $x_{1}^{*}=0.41317591116653, x_{2}^{*}=$ $0.96984631039295, x_{3}^{*}=0.11697777844051$, with $a=-8$. By Remark 6 , since $a_{3 c r}=27$, this orbit is stabilizable by the proposed scheme. In fact, simulations show that for the range $0.5<K<150000$, the stabilization is possible. In Fig. 2, we show simulation result of a typical case with $K=1$; initial condition is chosen as $x(0)=0.6$. In Fig. 2(a), we show $d\left(x(k), \Sigma_{3}\right)$ versus $k$, and as can be seen the decay is exponential. The $x(k-1)$ versus $x(k)$ plot in Fig. 2(b) is plotted for $k \geq 500$. As can be seen from these figures, the solutions converge to period 3 orbit characterized by $\Sigma_{3}$. The required input $u(k)$ given by (21) is given in Fig. 2(c). As can be seen,

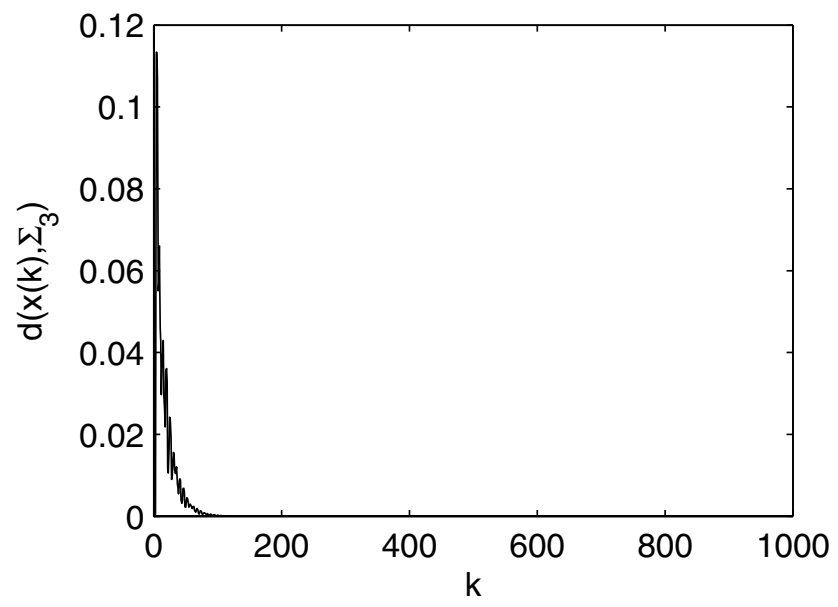

(a)

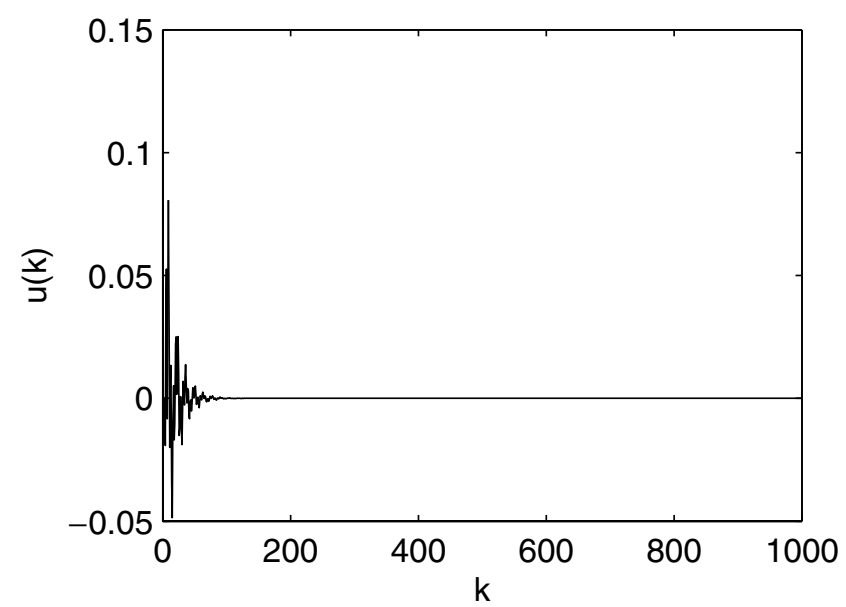

(c) $u(k)$ is quite small and $\rightarrow 0$ as $k \rightarrow \infty$. Finally Fig. 2(d) shows $x(k)$ versus $k$ for $980 \leq k \leq 1000$. Note that this orbit, in fact none of period 3 orbits of (33) with $r>3.88$, can be stabilized by DFC, see [Morgül, 2003].

Now let us choose $r=3.97$ and $T=4$. The system (33) has a period 4 orbit given by $\Sigma_{4}=\left\{x_{1}^{*} x_{2}^{*} x_{3}^{*} x_{4}^{*}\right\}$ with $x_{1}^{*}=0.28059766388136$, $x_{2}^{*}=0.80139458117555, x_{3}^{*}=0.63187038655891$, $x_{4}^{*}=0.92346249856075$ with $a=-14.676$. By Remark 6 , since $a_{4 c r}=15$, this orbit is stabilizable with the proposed scheme, and the critical gain given by (30) is $K_{c r}=0.4786$. In fact, simulations show that for the range $K_{c r}<K<0.546$, stabilization is possible. In Fig. 3, we show the simulation result of a typical case with $K=0.48$; initial

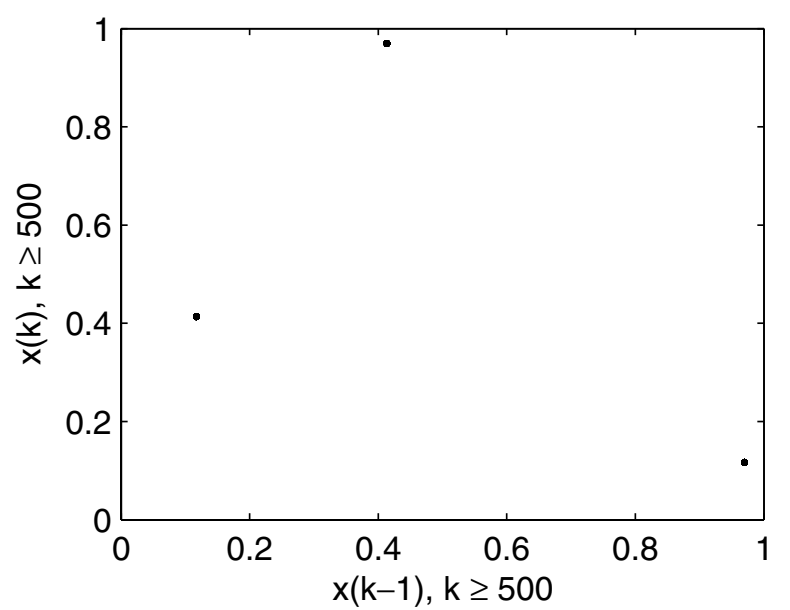

(b)

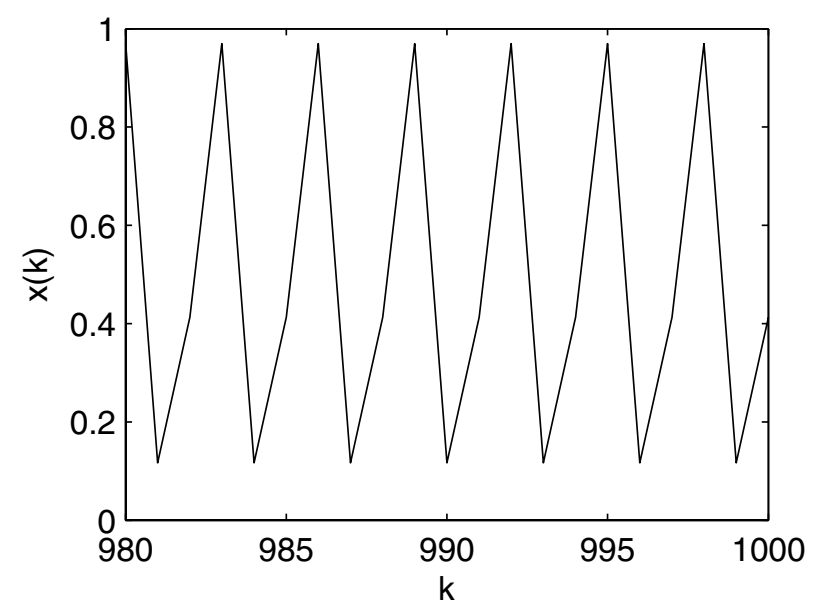

(d)

Fig. 2. Proposed scheme applied to logistic map, $r=4, T=3$, (a) $d\left(x(k), \Sigma_{3}\right)$ versus $k$, (b) $x(k-1)$ versus $x(k)$ for $k \geq 500$, (c) $u(k)$ versus $k,(\mathrm{~d}) x(k)$ versus $k$ for $980 \leq k \leq 1000$. 


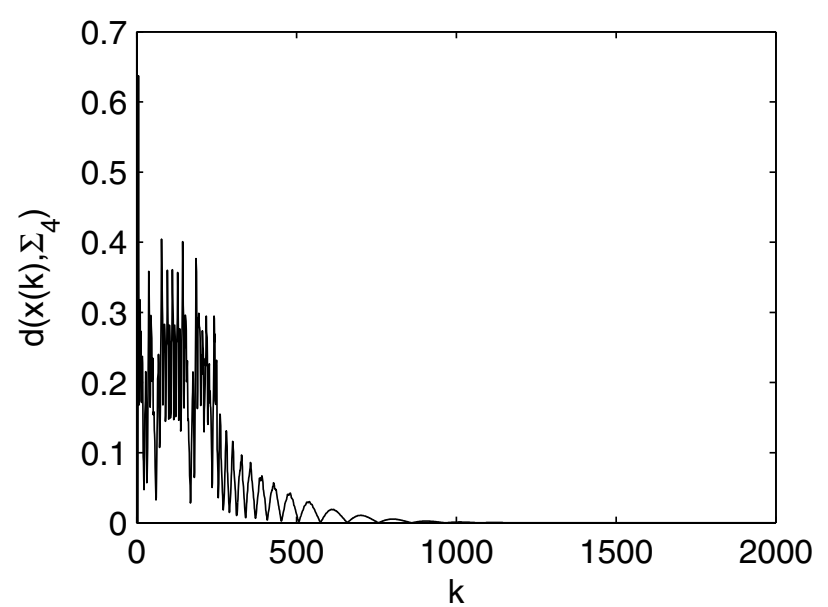

(a)

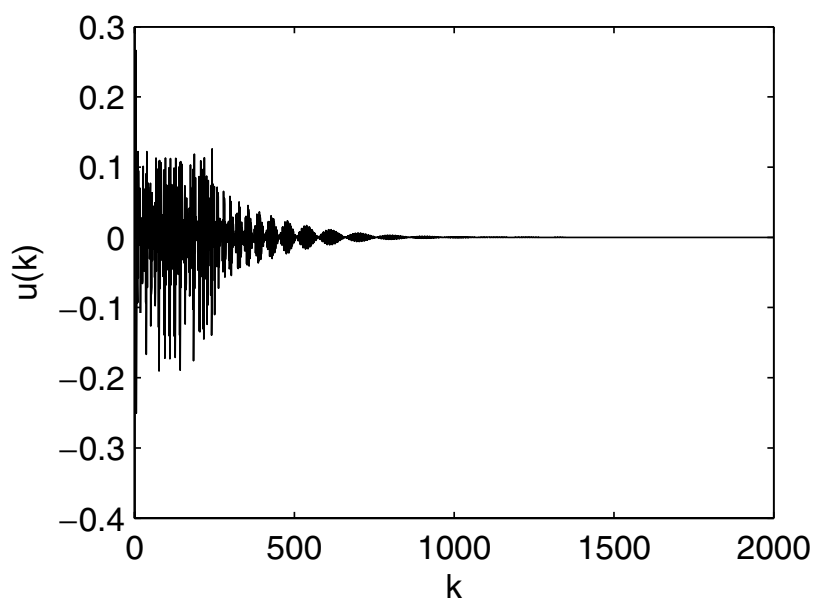

(c)

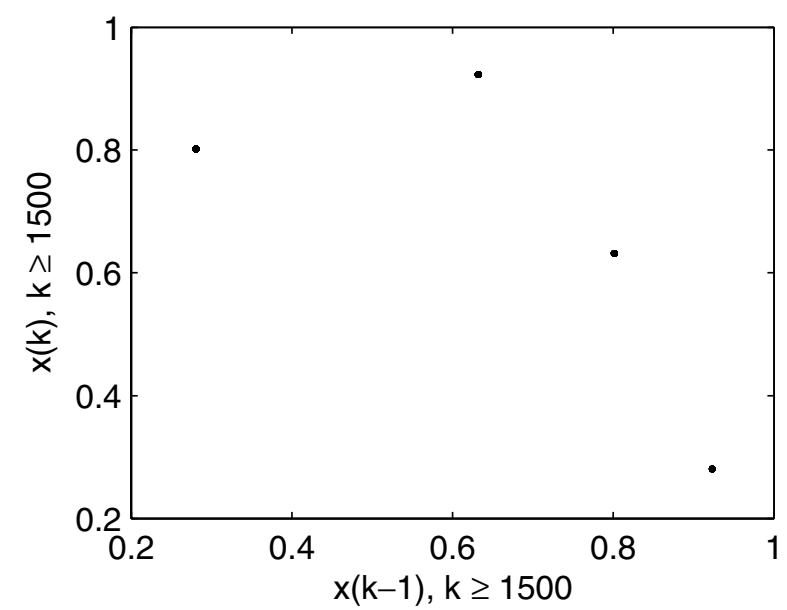

(b)

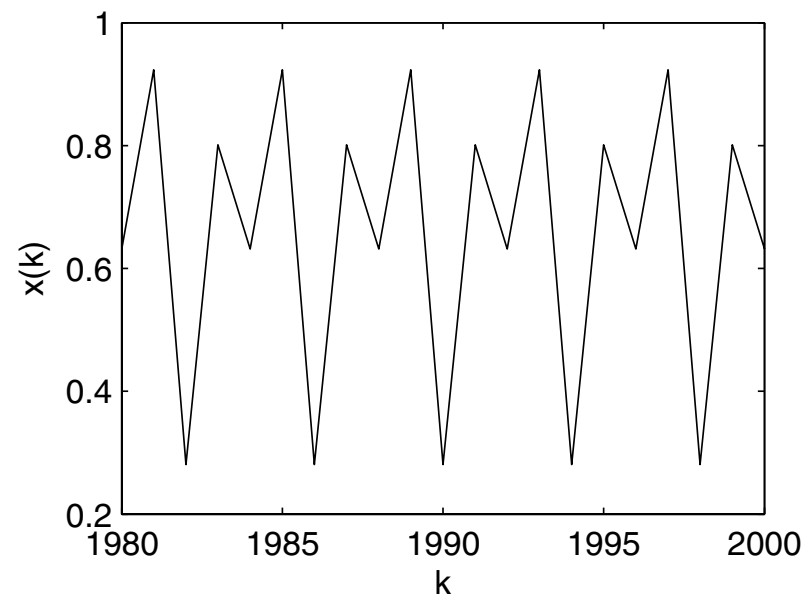

(d)

Fig. 3. Proposed scheme applied to logistic map, $r=3.97, T=4$, (a) $d\left(x(k)\right.$, $\left.\Sigma_{4}\right)$ versus $k$, (b) $x(k-1)$ versus $x(k)$ for $k \geq 1500$, (c) $u(k)$ versus $k$, (d) $x(k)$ versus $k$ for $1980 \leq k \leq 2000$.

condition is chosen as $x(0)=0.4$. In Fig. 3(a), we show $d\left(x(k), \Sigma_{4}\right)$ versus $k$, and as can be seen the decay is exponential. The $x(k-1)$ versus $x(k)$ plot in Fig. 3(b) is plotted for $k \geq 1500$. As can be seen from these figures, the solutions converge to period 4 orbit characterized by $\Sigma_{4}$. The required input $u(k)$ given by (21) is given in Fig. 3(c). As can be seen, $u(k)$ is quite small and $\rightarrow 0$ as $k \rightarrow \infty$. Finally Fig. $3(\mathrm{~d})$ shows $x(k)$ versus $k$ for $1980 \leq k \leq 2000$.

Now let us choose $r=3.75$ and $T=5$. The system (33) has a period 5 orbit given by $\Sigma_{5}=\left\{x_{1}^{*} x_{2}^{*} x_{3}^{*} x_{4}^{*} x_{5}^{*}\right\}$ with $x_{1}^{*}=0.93597631662827$, $x_{2}^{*}=0.22471744252216, x_{3}^{*}=0.65332317580673$, $x_{4}^{*}=0.84934501410202, x_{5}^{*}=0.47984272920771$ with $a=-3.0749$. By Remark 6, since $a_{5 c r}=11.5$, this orbit is stabilizable with the proposed scheme, and the critical gain given by (30) is $K_{c r}=0.1259$. In fact, simulations show that for the range $K_{c r}<$ $K<11$, the stabilization is possible. In Fig. 4, we show the simulation result of a typical case with $K=1$; initial condition is chosen as $x(0)=0.5$. In Fig. 4(a), we show $d\left(x(k), \Sigma_{5}\right)$ versus $k$, and as can be seen the decay is exponential. The $x(k-1)$ versus $x(k)$ plot in Fig. 4(b) is plotted for $k \geq 500$. As can be seen from these figures, the solutions converge to period 5 orbit characterized by $\Sigma_{5}$. The required input $u(k)$ given by $(21)$ is given in Fig. 4(c). As can be seen, $u(k)$ is quite small and $\rightarrow 0$ as $k \rightarrow \infty$. Finally Fig. 4(d) shows $x(k)$ versus $k$ for $980 \leq k \leq 1000$.

Finally, let us choose $r=3.65$ and $T=6$. The system (33) has a period 6 orbit given by $\Sigma_{6}=$ 


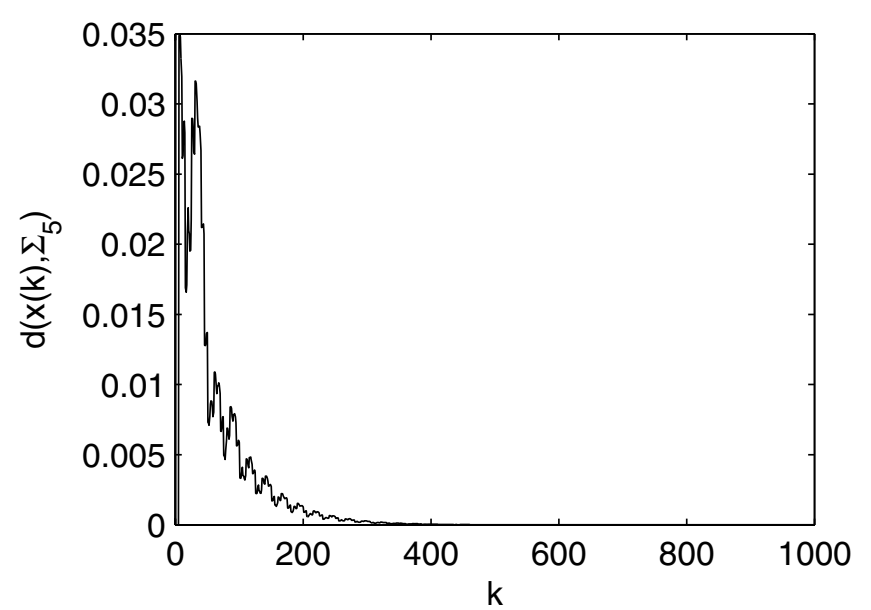

(a)

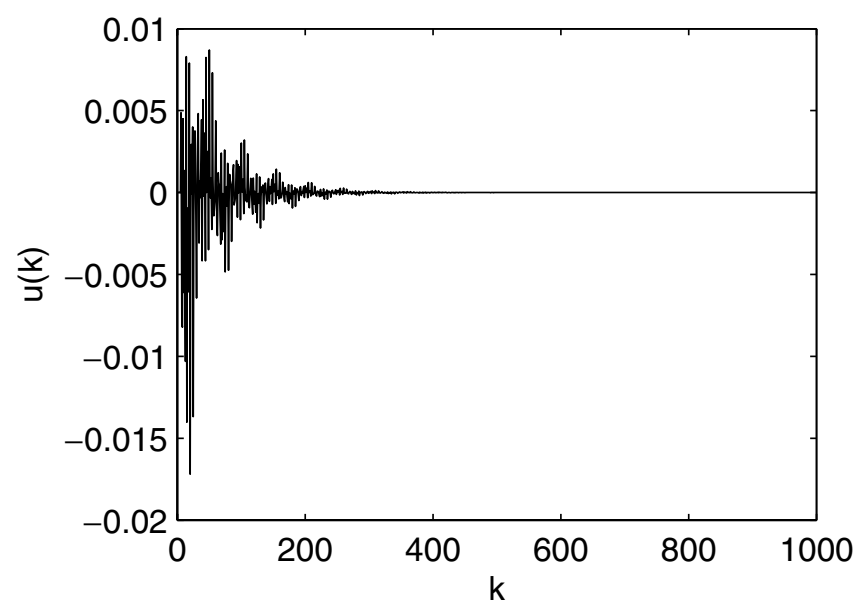

(c)

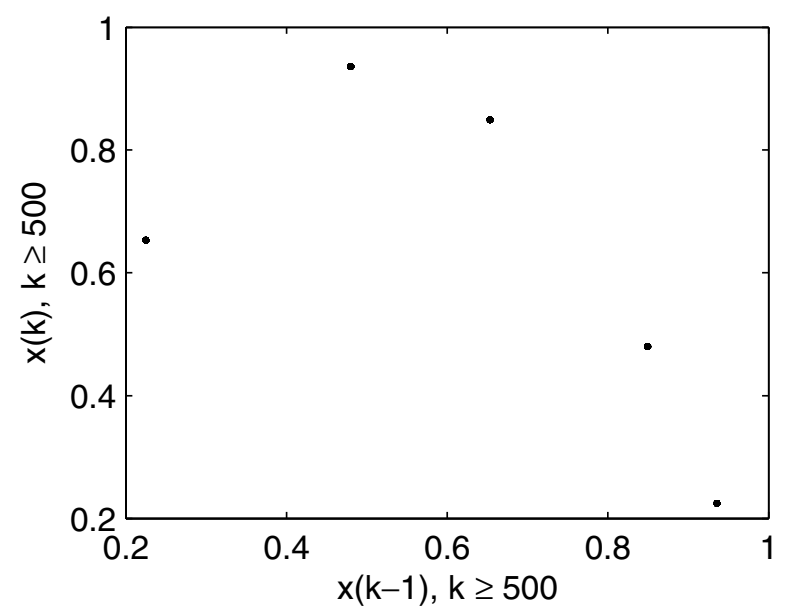

(b)

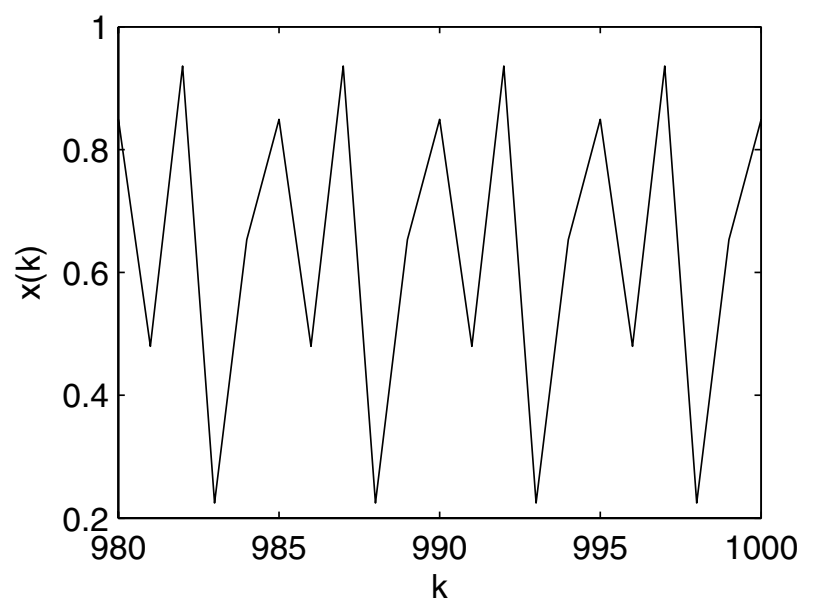

(d)

Fig. 4. Proposed scheme applied to logistic map, $r=3.75, T=5$, (a) $d\left(x(k), \Sigma_{5}\right)$ versus $k$, (b) $x(k-1)$ versus $x(k)$ for $k \geq 500$, (c) $u(k)$ versus $k$, (d) $x(k)$ versus $k$ for $980 \leq k \leq 1000$.

$\left\{x_{1}^{*} x_{2}^{*} x_{3}^{*} x_{4} x_{5}^{*} x_{6}^{*}\right\}$ with $x_{1}^{*}=0.52704092694303, x_{2}^{*}=$ $0.90983107718572, x_{3}^{*}=0.29944048183068, x_{4}^{*}=$ $0.76568196080167, x_{5}^{*}=0.65485779932172, x_{6}^{*}=$ 0.82496957626070 with $a=-4.4972$. By Remark 6 , since $a_{6 c r}=9.8$, this orbit is stabilizable with the proposed scheme, and the critical gain given by (30) is $K_{c r}=0.1423$. In fact, simulations show that for the range $K_{c r}<K<0.846$, stabilization is possible. In Fig. 5, we show simulation result of a typical case with $K=0.15$; initial condition is chosen as $x(0)=0.52$. In Fig. $5(\mathrm{a})$, we show $d\left(x(k), \Sigma_{6}\right)$ versus $k$, and as can be seen the decay is exponential. The $x(k-1)$ versus $x(k)$ plot in Fig. $5(\mathrm{~b})$ is plotted for $k \geq 500$. As can be seen from these figures, the solutions converge to period 6 orbit characterized by $\Sigma_{6}$. The required input $u(k)$ given by $(21)$ is given in Fig. 5(c). As can be seen, $u(k)$ is quite small and $\rightarrow 0$ as $k \rightarrow \infty$. Finally Fig. 5(d) shows $x(k)$ versus $k$ for $970 \leq k \leq 1000$.

One may argue that for higher order periodic solutions stabilization may become increasingly difficult, and after some period number, may become impossible for the proposed method. Clearly, not all periodic solutions can be stabilized with the proposed algorithm; however some of the higher order periodic solutions can still be stabilized with the proposed scheme. This apparently depends crucially on $a$ and $a_{m c r}$. We also considered (33) with $r=$ 3.65 , and find a period 10 solution, with $a=-7.74$, and it turned out that this orbit can be stabilized with the gain $K_{c r}=0.1135<K<0.151$. We have also considered the case $r=3.57$ and find a period 


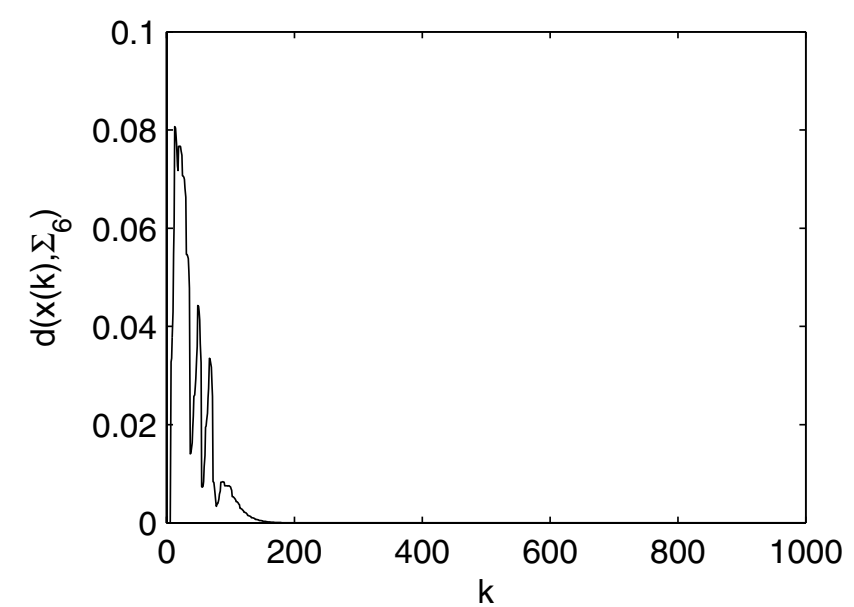

(a)

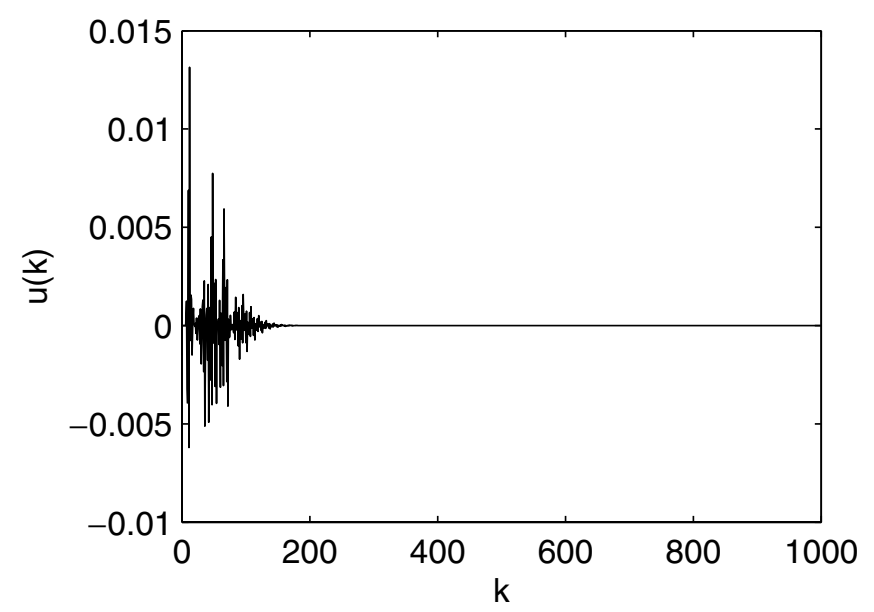

(c)

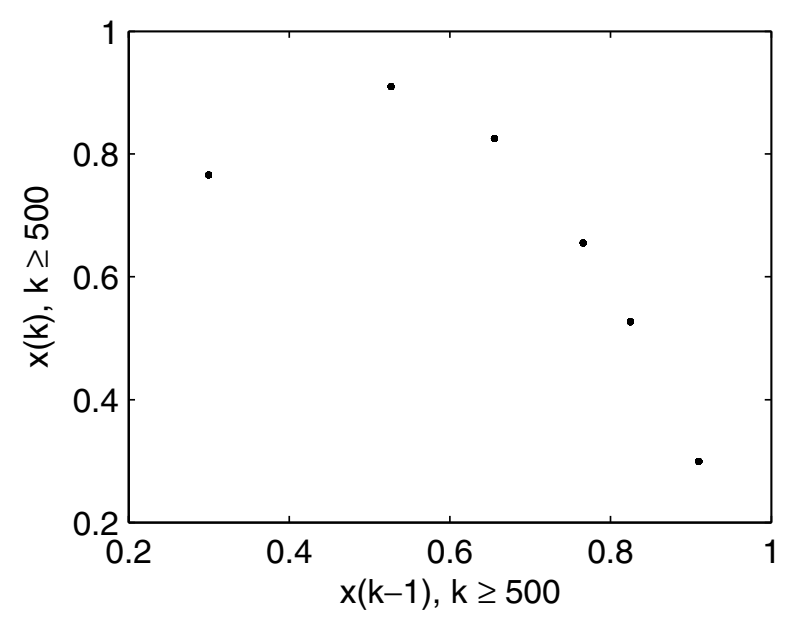

(b)

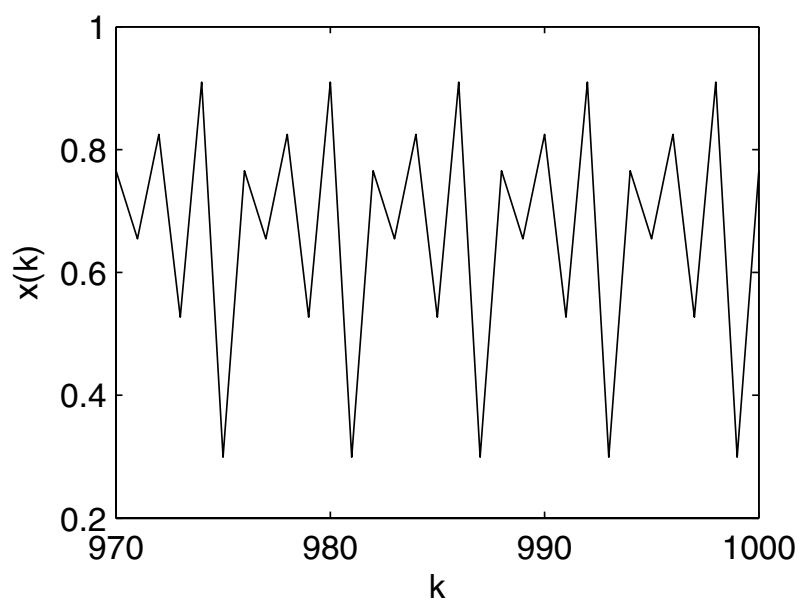

(d)

Fig. 5. Proposed scheme applied to logistic map, $r=3.65, T=6$, (a) $d\left(x(k)\right.$, $\left.\Sigma_{6}\right)$ versus $k$, (b) $x(k-1)$ versus $x(k)$ for $k \geq 500$, (c) $u(k)$ versus $k$, (d) $x(k)$ versus $k$ for $970 \leq k \leq 1000$.

16 solution with $a=-1.629$, and it turned out that this orbit can also be stabilized with the gain $K_{c r}=0.0155<K<1.455$.

\section{Conclusion}

In this letter we have considered the problem of stabilization of unstable periodic orbits of onedimensional discrete-time chaotic systems. Our approach is related to that of [Vieira \& Lichtenberg, 1996] for $T=1$. However, the form of our proposed control law is different for higher order periodic orbits and the extension to case $T>1$ is novel. We show that for $T=1$, the proposed scheme does not have the inherent limitations of DFC, however, for case $T>1$, the same limitation is also valid as for our scheme as well. However, we show that still many periodic orbits which cannot be stabilized by the DFC can be stabilized by the proposed scheme. In particular, for $T=1$ any hyperbolic periodic orbit, and for $T=2$ any orbit with $a<-1$ can be stabilized by the proposed scheme. Following a technique used in [Morgül, 2003, 2005a], we first constructed a map whose fixed points correspond to the periodic orbits of the uncontrolled system. Then we studied the stability of the proposed scheme by using the constructed map. We found the Jacobian of the constructed map at the periodic orbit in question and obtained its characteristic polynomial, which has a very simple structure. Then the stability problem is reduced to studying the Schur stability of the characteristic polynomial in question. We also presented some simulation results. 
Various generalizations of the proposed scheme can be done. First, the proposed scheme can be generalized to higher dimensions. Since for $T=1$ the proposed scheme does not have a serious limitation for stabilization, we may combine the proposed scheme with a possible generalization of periodic feedback to stabilize periodic orbits with $T>1$ without serious limitations, see e.g. [Schuster \& Stemmler, 1997; Morgül, 2005b, 2006].

\section{References}

Bleich, M. E. \& Socolar, J. E. S. [1996] "Stability of periodic orbits controlled by time delay feedback," Phys. Lett. A 210, 87-94.

Boccaletti, S., Grebogi, C., Lai, Y. C., Mancini, H. \& Maza, D. [2000], "The control of chaos: Theory and applications," Phys. Rep. 329, 103-197.

Chen, G. \& Dong, X. [1998] From Chaos to Order: Methodologies, Perspectives and Applications (World Scientific, Singapore).

Devaney, R. L. [1987] Chaotic Dynamical Systems (Addison-Wesley, Redwood City).

Elaydi, S. N. [1996] An Introduction to Difference Equations (Springer-Verlag, NY).

Fiedler, B., Flunkert, V., Georgi, M., Hövel, P. \& Schöll, E. [2007] "Refuting the odd-number limitation of time-delayed feedback control," Phys. Rev. Lett. 98, 114110.

Fradkov, A. L. \& Evans, R. J. [2005] "Control of chaos: Methods and applications in engineering," Ann. Rev. Contr. 29, 33-56.

Fuh, C. C. \& Tung, P. C. [1995] "Controlling chaos using differential geometric method," Phys. Rev. Lett. 75, 2952-2955.

Harrington, I. \& Socolar, E. S. [2004] "Design and robustness of delayed feedback controllers for discrete systems," Phys. Rev. E 69, 1200-1207.

Hino, T., Yamamoto, S. \& Ushio, T. [2002] "Stabilization of unstable periodic orbits of chaotic discrete time systems using prediction based feedback control," Int. J. Bifurcation and Chaos 12, 439-446.

Höhne, K., Shirahama, H., Choe, C. U., Benner, H. \& Pyragas, K. [2007] "Global properties in an experimental realization of time-delayed feedback control with an unstable control loop," Phys. Rev. Lett. 75, 214102.

Ishii, M., Konishi, K. \& Kokame, H. [1997] "Robust stability of extended delayed-feedback control in onedimensional chaotic systems," Phys. Lett. A 235, 603609.

Khalil, H. K. [2002] Nonlinear Systems, 3rd edition (Prentice-Hall, Upper Saddle River).

Kittel, A., Parisi, J. \& Pyragas, K. [1995] "Delayed feedback control of chaos by self-adapted delay time," Phys. Lett. A 198, 433-436.
Liu, R. H. \& Tan, W. H. [1998] "Nonlinear control of chaos," Chinese Phys. Lett. 15, 249-251.

Morgül, Ö. [2003] "On the stability of delayed feedback controllers," Phys. Lett. A 314, 278-285.

Morgül, Ö. [2005a] "On the stability of delayed feedback controllers for discrete time systems," Phys. Lett. A 335, 31-42.

Morgül, Ö. [2005b] "On the stabilization of periodic orbits for discrete time chaotic systems," Phys. Lett. A 335, 127-138.

Morgül, Ö. [2006] "Stabilization of unstable periodic orbits for discrete time chaotic systems by using periodic feedback," Int. J. Bifurcation and Chaos 16, 311323.

Nakajima, H. [1997] "On analytical properties of delayed feedback control of chaos," Phys. Lett. A 232, 207210.

Nakajima, H. \& Ueda, Y. [1998a] "Half period delayed feedback control for dynamical systems with symmetries," Phys. Rev. E 55, 1757-1763.

Nakajima, H. \& Ueda, Y. [1998b] "Limitation of generalized delayed feedback control," Physica D 111, 143-150.

Ott, E., Grebogi, C. \& Yorke, J. A. [1990] "Controlling chaos," Phys. Rev. Lett. 64, 1196-1199.

Pyragas, K. [1992] "Continuous control of chaos by self controlling feedback," Phys. Lett. A 170, 421-428.

Pyragas, K. [2001] "Control of chaos via an unstable delayed feedback controller," Phys. Rev. Lett. 86, 2265-2268.

Schuster, H. G. \& Stemmler, M. B. [1997] "Control of chaos by oscillating feedback," Phys. Rev. E 56, 64106417.

Solak, E., Morgül, Ö. \& Ersoy, U. [2001] "Observer-based control of a class of chaotic systems," Phys. Lett. A 279, 47-55.

Ushio, T. [1996] "Limitation of delayed feedback control in nonlinear discrete time systems," IEEE Trans. Circuits Syst.-I 43, 815-816.

Ushio, T. \& Yamamoto, S. [1998] "Delayed feedback control with nonlinear estimation in chaotic discrete-time systems," Phys. Lett. A 247, 112-118.

Ushio, T. \& Yamamoto, S. [1999] "Prediction based control of chaos," Phys. Lett. A 264, 30-35.

Vieira, D. S. M. \& Lichtenberg, A. J. [1996] "Controlling chaos using nonlinear feedback with delay," Phys. Rev. E 54, 1200-1207.

Zhu, J. \& Tian, Y. P. [2005a] "Stabilizing periodic solutions of nonlinear systems and applications in chaos control," IEEE Trans. Circuits Syst.-II 52, 870-874.

Zhu, J. \& Tian, Y. P. [2005b] "Necessary and sufficient conditions for stability of discrete-time systems via delayed feedback control," Phys. Lett. A 343, 95-107.

Zhu, J. \& Tian, Y. P. [2006] "Stabilization of unstable periodic solutions by nonlinear recursive delayed feedback control," Int. J. Bifurcation and Chaos 16, 29352947. 